\title{
Hypersensitivitätssyndrom nach Einnahme von Carbamazepin
}

Hypersensitivity Syndrome During Medication with Carbamazepine

G. Wagner

Zusammenfassung

Bei einer 51-jährigen Patientin mit bekannter endogener Depression entwickelte sich 4 Wochen nach Einleitung einer Carbamazepin-Therapie ein generalisiertes makulo-papulöses Exanthem. Neben septisch verlaufenden Temperaturen, einer Splenomegalie und einer generalisierten Lymphadenopathie zeigten sich deutliche Erhöhungen der Transaminasen und eine ausgeprägte Eosinophilie. Nach Abbruch der Carbamazepin-Therapie und systemischer Behandlung mit Kortikosteroiden kam es zu einer vollständigen Rückbildung der klinischen und laborchemischen Symptomatik.

Das hier vorgestellte Hypersensitivitätssyndrom muss zur Gruppe der potenziell lebensbedrohlichen Arzneimittelreaktionen gezählt werden. Dabei ist die Prognose von einer frühzeitig einsetzenden, hochdosierten Behandlung mit Kortikosteroiden abhängig. Vor diesem Hintergrund ist die differenzialdiagnostische Abgrenzung des Hypersensitivitätssyndroms von klinisch ähnlich verlaufenden Virusinfektionen zwingend notwendig.

\section{Abstract}

After a four weeks period of taking carbamazepine for treatment of endogenous depression a 51 years old female patient developed a widespread eruption of erythematous macules and papules. Additionally the patient showed septic temperatures, splenomegaly, a pronounced lymphadenopathy and a clear increase of transaminases and eosinophilic leucocytes. When therapy with carbamazepine was stopped and after treatment with systemic steroids clinical symptoms and laboratory parameter completely resolved.

A hypersensitivity syndrome has to be taken into account as a potential life threatening drug eruption. The prognosis depends on early onset of steroid medication. Therefore virus infections should urgently be considered as a differential diagnosis.

\section{Einleitung}

Das medikamentös ausgelöste Hypersensitivitätssyndrom, das auch mit dem Akronym DRESS für Drug Related Eosinophilia with Systemic Symptoms beschrieben wird, ist eine klinisch häufig bedrohlich verlaufende, zahlreiche Organsysteme betreffende Arzneimittelreaktion [1]. Dabei erfüllt das Hypersensitivitätssyndrom die Kriterien der Idiosynkrasie. Hierzu zählen das Auftreten der klinischen Symptomatik frühestens eine Woche nach Beginn der Arzneimitteleinnahme bzw. innerhalb von 24 Stunden bei Reexposition. Zu den typischen Symptomen zählen Fieber, Exantheme, eine meist ausgeprägte Eosinophilie und eine
Beteiligung innerer Organe, wobei typischerweise die Leber und das hämatopoetische System betroffen sind [2]. Pathogenetisch wird bei einer Idiosynkrasiereaktion ein individueller, genetisch determinierter Faktor postuliert, der einzelne Patienten prädisponiert, bei Einnahme bestimmter Arzneimittel in der beschriebenen Form zu reagieren [3]. Das Hypersensitivitätssyndrom kann in jedem Alter auftreten und ist auch bei Kindern wiederholt beschrieben worden [4,5]. Die Geschlechtsverteilung ist ausgeglichen [6,7]. Neben der Haut werden bei Hypersensitivitätssyndromen vor allem das lymphatisch-hämatologische System, die Leber und die Nieren, seltener die Lungen, das Herz oder das Zentralnervensystem in den Krankheitsprozess einbe- 
zogen. Die Letalität des Hypersensitivitätssyndroms, die mit $8-10 \%$ angegeben wird, ist in den meisten Fällen auf eine fulminant verlaufende Hepatitis zurückzuführen [8,9]. Zu den auslösenden Arzneimitteln zählen hauptsächlich aromatische Antikonvulsiva, Antibiotika und reverse Transkriptase-Inhibitoren [7,10 - 12]. Dabei werden die aromatischen Antikonvulsiva Carbamazepin und Phenytoin mit Abstand am häufigsten für die Entwicklung eines Hypersensitivitätssyndroms verantwortlich gemacht. Von 17 Patienten, die Bork mit einem Hypersensitivitätssyndrom beobachten konnte, hatten 12 Patienten Carbamazepin und die übrigen Phenytoin eingenommen [6]. Insgesamt muss bei den aromatischen Antikonvulsiva mit einem Hypersensitivitätssyndrom bei 5000 behandelten Patienten gerechnet werden [6]. Weitere Arzneimittel, die gehäuft zum Auftreten von Hypersensitivitätssyndromen führen können, sind innerhalb der Gruppe der Antibiotika Minocyclin und die Sulfonamide einschließlich Diaminodiphenylsulfon (DADPS) sowie Abacavir, Nevirapin und Zalcitabin als Vertreter der reversen TranskriptaseInhibitoren [7,10-12]. In Einzelfällen wurden Hypersensitivitätssyndrome auch durch Allopurinol, Goldsalze und Azathioprin ausgelöst $[6,13]$.

Der Fall der hier vorgestellten Patientin ist in seinem Verlauf und im Auftreten der klinischen und laborchemischen Befunde exemplarisch für ein Hypersensitivitätssyndrom nach Einnahme von Carbamazepin. Auch die zunächst bestehenden Probleme in der differentialdiagnostischen Abgrenzung von akuten Virusinfektionen sind für das Hypersensitivitätssyndrom typisch und unterstreichen die Notwendigkeit der Kenntnis dieser bedrohlich verlaufenden Arzneimittelreaktion.

\section{Kasuistik}

\section{Anamnese}

Bei der jetzt 52-jährigen Patientin war vor etwa 30 Jahren eine endogene Depression aufgetreten, die seither in unregelmäßigen Abständen episodenhaft rezidivierte. Bei einem entsprechenden Bedarf waren in den letzten Jahren Behandlungen mit tri- oder tetrazyklischen Antidepressiva, MAO-Hemmern oder selektiven Serotonin-Wiederaufnahmehemmern durchgeführt worden. Bei einer erneuten Zunahme der depressiven Symptomatik hatte die Patientin nun erstmals Carbamazepin in einer täglichen Dosierung von $200 \mathrm{mg}$ verordnet bekommen. Vier Wochen nach Einleitung dieser Therapie entwickelten sich Halsschmerzen, Schluckbeschwerden und Temperaturen bis $40^{\circ} \mathrm{C}$. Gleichzeitig wurden Lymphknotenschwellungen bemerkt und ein an Ausbreitung schnell zunehmendes Exanthem. Eine daraufhin eingeleitete Behandlung mit einem Penicillin-Präparat hatte keinerlei Einfluss auf die klinische Symptomatik, so dass die Patientin bei einer weiteren Verschlechterung ihres Allgemeinbefindens stationär aufgenommen werden musste.

\section{Aufnahmebefund}

Am Stamm, proximal an den Extremitäten, am Hals sowie im Bereich des Gesichtes fand sich ein makulo-papulöses, zu größeren Flächen konfluierendes Exanthem (Abb.1 u. 2). Darüber hinaus zeigte sich ein ausgeprägt ödematöses Erythem der Mund- und Rachenschleimhaut.

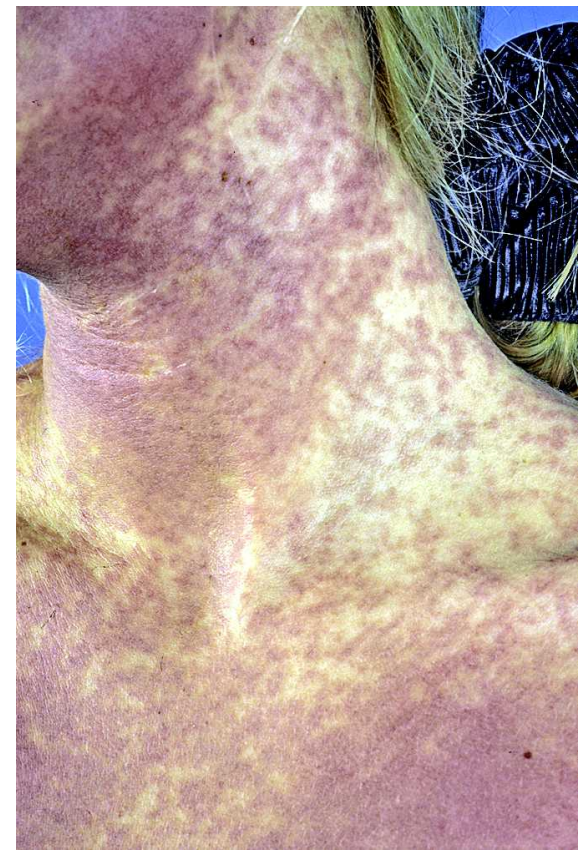

Abb. 1 Makulo-papulöses Exanthem Gesicht, Hals und vorderes Dekolleté.

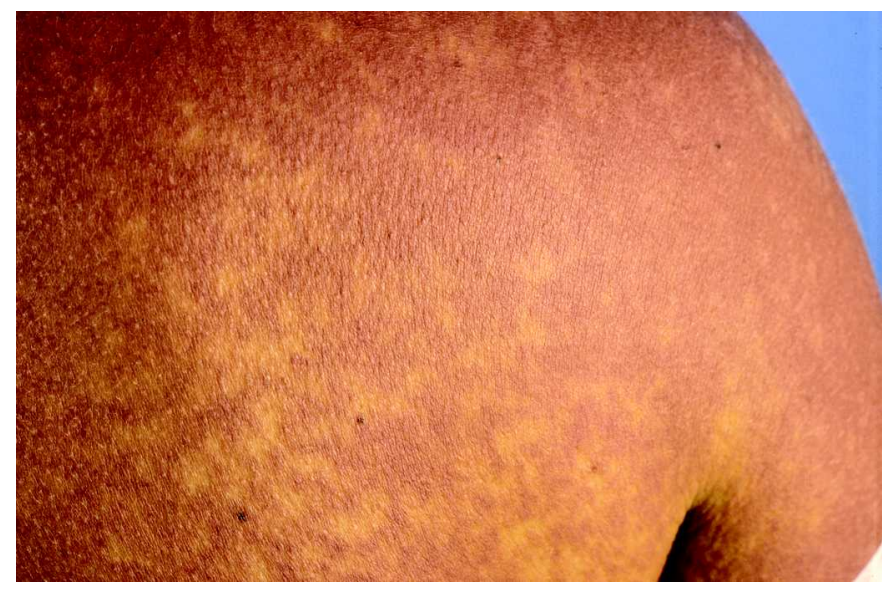

Abb. 2 Zu größeren Flächen konfluierender Befund am Rücken.

Temperatur bei Aufnahme $39,2^{\circ} \mathrm{C}$. Zervikal, axillär und inguinal konnten druckdolente Lymphknoten getastet werden. Das Allgemeinbefinden der Patientin war insgesamt deutlich reduziert.

\section{Histopathologische Befunde}

Perivaskuläres und interstitielles entzündliches Infiltrat in der verbreiterten papillären Dermis, das auf die angrenzende Epidermis übergreift und aus Lymphozyten, Histiozyten und vereinzelten eosinophilen Granulozyten besteht (Abb. 3 u. 4; Dr. C. Diaz, Einsendungslabor für Dermatopathologie Freiburg).

\section{Laborbefunde}

Blutbild: Leukozyten 14.0/nl (NW: 4-10,5/nl). Erythrozyten, Hämoglobin, Hämatokrit und Thrombozyten in den jeweiligen Normbereichen. Diff.-BB: Stabkernige 10\% (NW: 0-6\%), Segmentkernige 50\% (NW: $45-85 \%$ ), Eosinophile 13\% (NW: 0-6\%), Basophile 1\% (NW: 0-3\%), Monozyten 9\% (NW: 1-11\%) und Lymphozyten 17\% (NW: 10-50\%). 


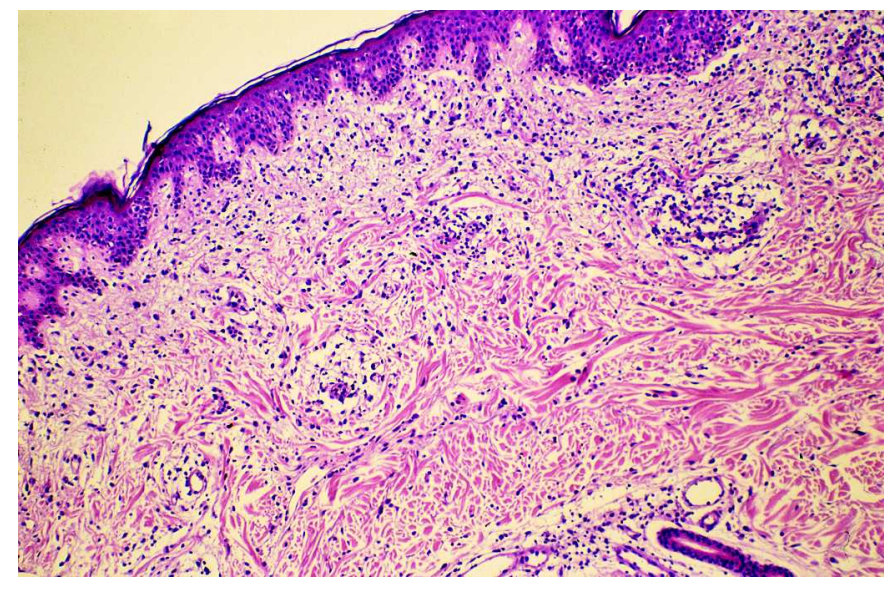

Abb. 3 Perivaskuläres und interstitielles Infiltrat in der papillären Dermis, auf die Epidermis übergreifend (HE × 100).

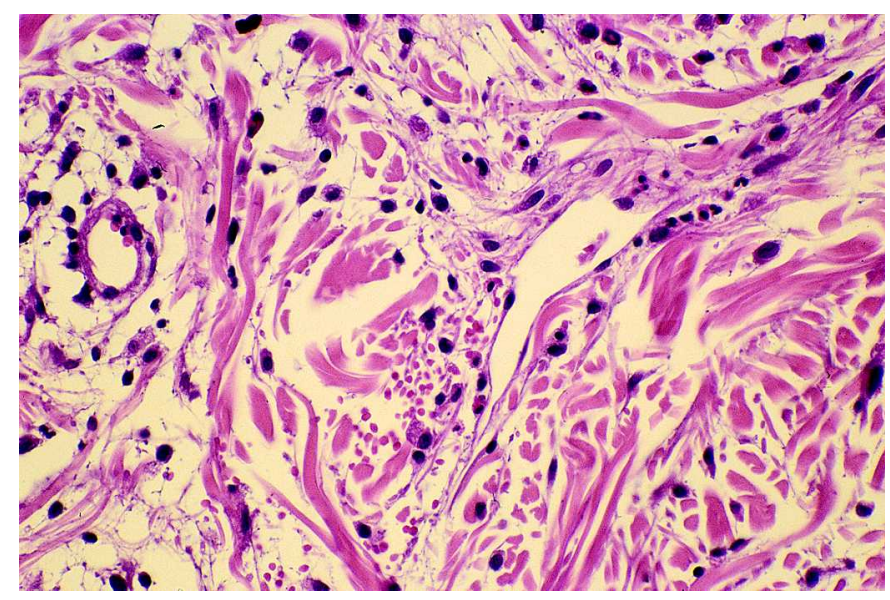

Abb. 4 In der ödematös aufgelockerten Dermis perivaskuläres lymphohistiozytäres Infiltrat mit einzelnen eosinophilen Granulozyten $(\mathrm{HE} \times 400)$.

Enzyme: GOT $121 \mathrm{U} / 1$ (NW: $0-32 \mathrm{U} / \mathrm{l})$, GPT $305 \mathrm{U} / \mathrm{l}$ (NW: 0-35U/1), gamma-GT $152 \mathrm{U} / \mathrm{l}(\mathrm{NW}: 0-38 \mathrm{U} / \mathrm{l})$, GLDH 12,1 U/1 (NW: <4,8U/1), APH $112 \mathrm{U} / 1$ (NW: 30-135U/1), LDH $473 \mathrm{U} / 1$ (NW: $126-253 \mathrm{U} / \mathrm{l})$ und CK $36 \mathrm{U} / \mathrm{l}(\mathrm{NW}:<185 \mathrm{U} / \mathrm{l}$ ).

Entzündungsparameter: BSG n. W. 32/76 mm (NW: 10/20 mm), CRP $27 \mathrm{mg} / \mathrm{l}$ (NW: 3-15 mg/l), Elektrophorese geringfügige Erhöhung der alpha-1- und alpha-2-Globulinfraktionen bei sonst unauffälligen Befunden.

Hepatitis-Serologie: Hep A-IgM-AK, Hbs-AG, Hep Bc-AK und Hep C-AK negativ.

Übrige Laborbefunde (ASL, Bilirubin, nierenpflichtige Substanzen, Gerinnungsparameter, Elektrolyte und Urinstatus) unauffällig.

\section{Ergänzende apparative Untersuchungen}

Die Röntgenaufnahme der Thoraxorgane und das EKG zeigten keine pathologischen Befunde.

In der Oberbauchsonographie konnte eine Splenomegalie bei sonst unauffälligen Befunden nachgewiesen werden. Die Lymph- knotensonographie zervikal, axillär und inguinal führte zum Nachweis einzelner bis zu 2,5 cm großer, physiologisch geschichteter Lymphknoten.

\section{Therapie und Verlauf}

Die klinische Symptomatik der Patientin konnte bei der stationären Aufnahme zunächst nicht sicher eingeordnet werden. Diskutiert wurden eine Virusinfektion, eine myeloproliferative Erkrankung und eine Arzneimittelreaktion. Durch laborchemische Untersuchungen konnten eine infektiöse Mononukleose sowie eine Hepatitis A, B und C ausgeschlossen werden. Auch fanden sich hierbei keine Hinweise für eine Leukämie oder ein Lymphom. Auffällig war hingegen eine Leukozytose, eine Eosinophilie und eine deutliche Erhöhung der Transaminasen. Bei den sonographischen Untersuchungen zeigten sich eine Splenomegalie und vergrößerte, morphologisch jedoch unauffällig geschichtete Lymphknoten. Der histopathologische Befund war durch eine Interface-Dermatitis mit Eosinophilie gekennzeichnet. Bei Wertung aller Befunde wurde die Diagnose eines Hypersensitivitätssyndroms gestellt. Nach Absetzen des Carbamazepins wurde eine orale Behandlung mit $100 \mathrm{mg}$ Prednisolon täglich eingeleitet, die bei schrittweiser Reduktion der Dosierung nach 2 Wochen beendet werden konnte. Zu diesem Zeitpunkt hatten sich das Arzneimittelexanthem und die Lymphadenopathie vollständig zurückgebildet. Auch die pathologischen Befunde im Blutund Differenzial-Blutbild waren nicht mehr nachweisbar. Die Normalisierung der Transaminasenwerte konnte hingegen erst weitere 3 Wochen später beobachtet werden. Die allergologischen Untersuchungen erfolgten 2 Monate nach Beendigung der oralen Prednisolon-Therapie. Bei der Epikutantestung mit Carbamazepin zeigte sich nach 48 und 72 Stunden ein deutlich infiltriertes Erythem. Der Lymphozyten-Transformationstest war hingegen negativ. Bei einer erneut aufgetretenen behandlungsbedürftigen Depression wurde der Patientin Nortriptylin verordnet, das ohne Nebenwirkungen vertragen wurde.

\section{Diskussion}

Arzneimittelexantheme nach Einnahme von Antikonvulsiva sind häufig und zeigen nicht selten eine ausgeprägte Manifestation. Bei Carbamazepin, einem der am häufigsten verordneten Antikonvulsiva, entwickeln 2-5\% der behandelten Patienten eine kutane Arzneimittelreaktion [14,15]. Kommt es darüber hinaus zu weiteren multisystemischen Nebenwirkungen, zu denen definitionsgemäß Fieber, eine Lymphadenopathie, eine Eosinophilie und die Erkrankung mindestens eines weiteren Organsystems gezählt werden, kann die Diagnose eines Hypersensitivitätssyndroms als gesichert angesehen werden [16]. Das Risiko, nach Einnahme von Carbamazepin ein Hypersensitivitätssyndrom zu entwickeln, ist jedoch insgesamt gering. Bei 10000 Verordnungen ist, Schätzungen zufolge, mit maximal 4 Erkrankungen zu rechnen [17].

Der Verlauf und die klinische Symptomatik eines Hypersensitivitätssyndroms sind grundsätzlich unabhängig von der im Einzelfall eingenommenen und für die Auslösung des Syndroms verantwortliche Arzneimittelsubstanz. Vereinzelt werden komplet- 
te und inkomplette Formen des Hypersensitivitätssyndroms unterschieden. Dabei gelten als Hauptsymptome Fieber, Exanthem, Lymphadenopathie und Hepatitis, deren gemeinsames Auftreten die komplette Form des Syndroms definiert, während bei einem inkompletten Hypersensitivitätssyndrom mindestens zwei der genannten vier Hauptsymptome vorliegen müssen [7]. Die Latenzzeit zwischen dem Beginn einer medikamentösen Therapie und dem Auftreten der ersten Symptome eines Hypersensitivitätssyndroms beträgt in den meisten Fällen 2-6 Wochen [13,18-20]. Nur sehr selten wurde über längere Latenzzeiten von 3 bis maximal 14 Monaten berichtet $[21,22]$.

Typischerweise ist die initiale Phase eines Hypersensitivitätssyndroms durch ein allgemeines Krankheitsgefühl, eine Laryngitis, Zephalgien, Arthralgien und Fieber gekennzeichnet. Die ebenfalls bereits in der Frühphase der Erkrankung auftretenden generalisierten Exantheme zeichnen sich durch eine deutliche klinische Polymorphie aus. Dabei werden makulo-papulöse, morbilliforme, rubeoliforme und pustulöse Formen beschrieben $[6,18,21,23]$. Purpuriforme und Erythema exsudativum multiforme-artige Exantheme, das Stevens-Johnson-Syndrom und die toxische epidermale Nekrolyse zählen ebenso wie lymphomartige Infiltrationen oder Papeln und Knoten zu den weiteren möglichen kutanen Arzneimittelreaktionen eines Hypersensitivitätssyndroms [6,22-25]. Nur in Ausnahmefällen wurde keine dermatologische Beteiligung beobachtet [26]. Die Lymphadenopathie als weiteres initiales Symptom manifestiert sich lokal im Bereich der peripheren Lymphknoten oder generalisiert peripher und intraabdominal. Histopathologisch zeigen die Lymphknoten eine benigne Hyperplasie bei sonst unauffälliger Struktur $[27,28]$. Der weitere Verlauf eines Hypersensitivitätssyndroms ist in meist zunehmender Weise durch die Mitbeteiligung viszeraler Organsysteme geprägt. Dabei ist die Leber mit 34-94\% das am häufigsten betroffene Organ [29,30]. Von besonderer Bedeutung ist die vereinzelt fulminant verlaufende Hepatitis, die als Hauptursache der mit 8-10\% hohen Letalität angesehen wird $[8,9]$. Das klinische Spektrum der meist anikterischen Hepatitiden umfasst eine asymptomatische Transaminasenerhöhung, eine deutliche Funktionseinschränkung mit Hepatomegalie und eine akut verlaufende Leberzellnekrose. Mit Ausnahme der fulminanten Verläufe zeigt sich das histopathologische Bild einer granulomatösen Hepatitis [23]. Neben der im Vordergrund stehenden Hepatitis sind die Myokarditis, die Pneumonie, die interstitielle Nephritis sowie die Thyreoiditis weitere internistische Manifestationen des Hypersensitivitätssyndroms. Auch eine zerebrale Symptomatik, eine Rhabdomyolyse und eine Splenomegalie wurden vereinzelt als zusätzliche Organbeteiligungen beobachtet [6,21,28,31-36]. Die für das Hypersensitivitätssyndrom typischen Laborbefunde sind Ausdruck einer primären Beteiligung des hämatologischen Systems oder können auf entzündliche Reaktionen der betroffenen Organsysteme zurückgeführt werden. Zu den am häufigsten auftretenden hämatologischen Störungen zählen die Eosinophilie, eine Leukozytose und eine teilweise mononukleose-artige atypische Lymphozytose, während Thrombozytopenien, Agranulozytosen oder hämolytische Anämien nur selten beschrieben worden sind [6,27,28,37]. Bei einer hepatogenen Manifestation des Hypersensitivitätssyndroms ist eine Erhöhung der Transaminasen obligat, während eine Hyperbilirubinämie selten und nur bei einzelnen Medikamenten, z.B. bei Diaminodiphenylsulfon, häufiger nachweisbar ist. Eine ausgeprägte Hyperbilirubinämie ist vermutlich mit einer schlechteren Prognose verbunden [7,28]. Bei einer Beteiligung anderer Organsysteme, z. B. bei einer Karditis, einer Niereninsuffizienz oder einer Thyreoiditis, finden sich richtungsweisende Laborbefunde.

Die Pathogenese des Hypersensitivitätssyndroms ist nicht geklärt. Als mögliche Ursache wird eine genetisch determinierte Störung in der Metabolisierung einzelner Arzneimittel diskutiert.

Der damit verbundene Anstieg toxischer oder immunologisch wirksamer Metaboliten wird für die klinische Symptomatik des Hypersensitivitätssyndroms verantwortlich gemacht. Die aromatischen Antikonvulsiva, zu denen Carbamazepin, Phenytoin, Primidon und Clonazepam zählen, werden durch die Cytochrom P-450-Isoenzyme hydroxiliert. Die entstehenden Zwischenprodukte, die Epoxide, gelten biologisch als besonders aktive Verbindungen. Ist deren weiterer enzymatischer Abbau behindert, z.B. durch eine Störung der Epoxidhydrase, kommt es zu einer Kumulation der Epoxide, die möglicherweise mit anderen Molekülen toxische oder immunologisch wirksame Verbindungen eingehen [38]. Als weiterer Hinweis für einen gestörten Metabolismus der aromatischen Antikonvulsiva gelten die bei einzelnen Patienten nachgewiesenen LKM-Antikörper (liver-kidney-microsomal antibody), die spezifisch gegen Cytochrom P-450-Isoenzyme gerichtet sind und für deren Funktionsstörung verantwortlich gemacht werden $[39,40]$. Einer anderen These zur Folge, die besonders in den letzten Jahren an Bedeutung gewonnen hat, ist die Reaktivierung der humanen Herpes-Viren 6 oder 7 ein wesentlicher Faktor in der Pathogenese des Hypersensitivitätssyndroms. Der Titeranstieg spezifischer IgG-Antikörper 3 bis 4 Wochen nach Auftreten der klinischen Symptome eines Hypersensitivitätssyndroms, der Nachweis der Virusreplikation im Serum vor Beginn der Antikörperproduktion und die direkt nachgewiesene Virus-DNA in erkrankten Organsystemen, z. B. im Liquor bei einer Enzephalitis, gelten als Belege für eine pathogenetische Bedeutung der Herpes-Viren [41-43].

Ein Hypersensitivitätssyndrom sicher zu diagnostizieren ist schwierig und wird besonders in der Anfangsphase der Erkrankung nur vereinzelt möglich sein. Dabei ist zu berücksichtigen, dass das Krankheitsbild selten und daher nur wenig bekannt ist, während die Diagnose klinisch gestellt werden muss. Darüber hinaus sind die klinischen und laborchemischen Befunde eines Hypersensitivitätssyndroms in ihrer Kombination so vielgestaltig und sicher auch ungewöhnlich, dass man in der Regel nicht sofort an eine Arzneimittelreaktion denken wird. Die Patienten sind im Allgemeinen schwer krank mit hohen Temperaturen, Zephalgien, Myalgien und den klinischen Zeichen eines Infektes der oberen Luftwege. Gleichzeitig werden neben einer Lymphadenopathie klinisch-morphologisch sehr unterschiedliche Exantheme beobachtet, die makulo-papulöse, morbilliforme oder auch rubeoliforme Manifestationen beinhalten können. Die klinische Symptomatik ist daher mit viralen oder auch bakteriellen Infektionen durchaus vereinbar, so dass in der Literatur zahlreiche Infektionskrankheiten als typische Differenzialdiagnosen zum Hypersensitivitätssyndrom angegeben werden. Einzelne Beispiele sind die infektiöse Mononukleose, der akute grippale Infekt, virale Hepatitiden, HIV-Infektionen, Masern, Röteln oder 
bakterielle Septikämien und das toxische StaphylokokkenSchocksyndrom [7,12,13,18,23]. Zu den weiteren Differenzialdiagnosen nicht infektiöser Genese zählt der Lupus erythematodes, das Hypereosinophilie-Syndrom und verschiedene Formen der Vaskulitiden. Bei Erwachsenen mit lymphomartigen Infiltrationen der Haut sind bei gleichzeitigem Nachweis einer Lymphadenopathie und schwer gestörtem Allgemeinbefinden der Patienten akute Lymphome differenzialdiagnostisch $\mathrm{zu}$ berücksichtigen, während bei Kindern in Einzelfällen auch ein mukokutanes lymphonodales Syndrom ausgeschlossen werden muss $[6,12,25,44]$. In Ergänzung zu der klinisch zu stellenden Diagnose eines Hypersensitivitätssyndroms können bei einzelnen Arzneimitteln, z. B. bei Carbamazepin, positive Epikutan- oder Lymphozyten-Transformationstestungen den kausalen Zusammenhang zwischen dem Syndrom und dem auslösenden Medikament sichern [45].

Die Behandlung eines Hypersensitivitätssyndroms besteht zunächst im Absetzen aller für die Auslösung des Syndroms in Frage kommenden Arzneimittel. Von wenigen Ausnahmen abgesehen, vertreten die meisten Autoren die Auffassung, dass bei einem Hypersensitivitätssyndrom die Indikation zu einer systemischen Therapie mit Kortikosteroiden gegeben ist, wobei Dosierungen von 100-150 mg oder 1-2 mg/kg Körpergewicht Prednisolon täglich empfohlen worden sind. Die Behandlung sollte über einen Zeitraum von 2-3 Wochen erfolgen, da ein schnelles Absetzen oder auch eine schnelle Reduktion der Dosis zu einem erneuten Auftreten der Symptomatik führen können $[12,13,22,23,27]$. Durch die Therapie mit Kortikosteroiden kommt es im Allgemeinen zu einer schnellen Rückbildung der klinischen Symptomatik einschließlich der dermatologischen Manifestationen, während sich bei den Laborbefunden insbesondere die Transaminasen erst nach 4 bis 6 Wochen normalisieren.

Nach einem durch Carbamazepin bedingten Hypersensitivitätssyndrom muss die neurologische Behandlung einer Epilepsie mit anderen Antikonvulsiva fortgeführt werden. Dabei sollten grundsätzlich keine aromatischen Antikonvulsiva verordnet werden, da Kreuzreaktionen aufgrund der ähnlichen Metabolisierungswege häufig sind $[46,47]$. Für die Therapie der betroffenen Patienten hat sich die Valproinsäure als Alternative bewährt $[19,47]$.

Die hier vorgestellte Patientin war wegen einer endogenen Depression mit Carbamazepin behandelt worden. Die Verordnung von tri- oder tetrazyklischen Antidepressiva ist bei einem Carbamazepin induzierten Hypersensitivitätssyndrom ebenfalls keineswegs unbedenklich, da Carbamazepin und zahlreiche Vertreter der genannten Antidepressiva chemisch gleiche oder ähnliche Ringstrukturen aufweisen [48]. Tatsächlich wurden vereinzelt Kreuzreaktionen zwischen Carbamazepin und trizyklischen Antidepressiva nachgewiesen [13]. Bei unserer Patientin stellte der behandelnde Psychiater die Indikation zur Therapie mit Nortriptylin, einem trizyklischen Antidepressivum, ohne dass Nebenwirkungen beobachtet werden konnten. Bei einer entsprechenden Indikation sollten Patienten mit einem bekannten Hypersensitivitätssyndrom sehr engmaschig klinisch und laborchemisch überwacht werden, um Kreuzreaktionen möglichst frühzeitig erkennen zu können.
Literatur

${ }^{1}$ Bocquet H, Bagot M, Roujeau JC. Drug-induced pseudolymphoma and drug hypersensitivity syndrome (Drug Rush with Eosinophilia and Systemic Symptoms: DRESS). Semin Cut Med Surg 1996; 15: 250-257 ${ }^{2}$ Uetrecht JP. The role of leukocyte-generated reactive metabolites in the pathogenesis of idiosyncratic drug reactions. Drug metabolism reviews 1992; 24: 299-366

${ }^{3}$ Coleman JW. Allergic reactions to drugs: current concepts and problems. Clin Exp Allergy 1990; 20: 79-85

${ }^{4}$ Bessmertny O, Hatton RC, Gonzalez-Peralta RP. Antiepileptic hypersensitivity syndrome in children. Ann Pharmacother 2001; 35: 533 538

${ }^{5}$ Verrotti A, Feliciani C, Morresi S et al. Carbamazepine-induced hypersensitivity syndrome in a child with epilepsy. Int J Immunpathol Pharmacol 2000; 13: 49-53

${ }^{6}$ Bork K. Arzneimittelnebenwirkungen an der Haut. 2. Aufl. Stuttgart, New York: Schatthauer, 1999

${ }^{7}$ Wozel G. Dapson. 1. Aufl. Stuttgart, New York: Thieme, 1996

${ }^{8}$ Parker WA, Shearer CA. Phenytoin hepatotoxicity. A case report and review. Neurology 1979; 29: 175-179

${ }^{9}$ Wolkenstein P, Revuz J. Drug-induced severe skin reactions. Incidence, management and prevention. Drug Safety 1995; 13: 56-68

${ }^{10}$ Brockmeyer NH. Deutsch-Österreichische Leitlinien zur antiretroviralen Therapie der HIV-Infektion. Dtsch Med Wochenschr 2003; 128: 7 18

${ }^{11}$ Kaufmann D, Pichler W, Beer J. Severe episode of high fever with rash, lymphadenopathy, neutropenia, and eosinophilia after minocycline therapy for acne. Arch Intern Med 1994; 154: 1983- 1984

${ }^{12}$ Knowles SR, Shapiro L, Shear NH. Serious adverse reactions induced bei minocycline. Arch Dermatol 1996; 132: 934-939

${ }^{13}$ Richter G, Horn K, Wozel G et al. Carbamazepin-Hypersensitivitätssyndrom. Z Klin Med 1986; 41: $1803-1804$

14 Sillanpää M. Carbamazepine: pharmacology and clinical uses. Acta Neurol Scand 1981; 64 (suppl 88): 1-202

${ }^{15}$ Zakrzewska JM, Ivanyi L. In vitro lymphocyte proliferation by carbamazepine, carbamazepine-10,11-epoxid and oxcarbamazepine in the diagnosis of drug-induced hypersensitivity. J Allergy Clin Immunol 1988; 82: $110-115$

${ }^{16}$ Schnyder B, Zanni MP, Pichler WJ. Medikamenteninduzierte Hypersensitivitätssyndrome. Schweiz Med Wschr 1997; 127: 355-359

17 Tennis P, Stern RS. Risk of serious cutaneous disorders after initiation of use of phenytoin, carbamezepine, or sodium valproate. A record linkage study. Neurology 1997; 49: 542-546

${ }^{18}$ Elstner S, Sperling W. Das Carbamazepin-Hypersensitivitäts-Syndrom. Fortschr Neurol Psychiat 2000; 68: 188-192

${ }^{19}$ Gall H, Merk H, Scherb W et al. Antikonvulsiva-HypersensitivitätsSyndrom auf Carbamazepin. Hautarzt 1994; 45: 494-498

20 Zeller A, Schaub N, Steffen I et al. Drug hypersensitivity syndrome to carbamazepine and human herpes virus 6 infection. Infection 2001; 31: $254-256$

${ }^{21}$ Kleier RS, Breneman DL, Boiko S. Generalized pustulation of an anticonvulsant hypersensitivity syndrome. Arch Dermatol 1991; 127: $1361-1364$

${ }^{22}$ Rütter A, Kalden H, Bonsmann G et al. Anticonvulsiva-Hypersensitivitäts-Syndrom. H u G 1998; 73: 741 - 744

${ }^{23}$ Ronsdorf A, Schlienger RG, Haefeli W et al. Antikonvulsiva Hypersensitivitäts-Syndrom. Schweiz Rundsch Med Prax 1999; 88: 1660 - 1666

${ }^{24}$ Bastuji-Garin S, Rzany B, Stern RS et al. Clinical classifications of cases of toxic epidermal necrolysis, Steven-Johnson syndrome, and erythema multiforme. Arch Dermatol 1993; 129: 92-96

${ }^{25}$ Schreiber MM, McGegor JG. Peudolymphoma syndrome. A sensitivity to anticonvulsant drugs. Arch Dermatol 1968; 97: 297-326

${ }^{26}$ Bonnetblanc JM. Drug hypersensitivity syndrome. Dermatology 1993; 187: $84-87$

${ }^{27}$ Chang D, Shear N. Cutaneous reactions to anticonvulsants. Sem Neurol 1992; 12: 329-337

${ }^{28}$ Vittorio CC, Muglia JJ. Anticonvulsant hypersensitivity syndrome. Arch Intern Med 1995; 155: 2285-2290

${ }^{29}$ Haruda R. Phenytoin hypersensitivity: 38 cases. Neurology 1979; 29: $1480-1485$

${ }^{30}$ Powers NG, Carson NH. Idiosyncratic reactions to phenytoin. Clin Pediatr 1987; 25: $120-124$ 
${ }^{31}$ Flowers FP, Aranjo OE, Hamm KA. Phenytoin hypersensitivity syndrome. J Emerg Med 1987; 5: $103-108$

32 Gupta A, Eggo MC, Uetrecht JP et al. Drug-induced hypothyroidism: The thyroid as a target organ in hypersensitivity reactions to anticonvulsants and sulfonamides. Clin Pharmacol Ther 1992; 51: 56-67

${ }^{33}$ Handfield-Jones SE, Jenkins RE, Whittaker SJ et al. The anticonvulsant hypersensitivity syndrome. Br J Dermatol 1993; 129: 175-177

${ }^{34}$ Ray-Chaudhuri K, Pye IF, Boggeld M. Hypersensitivity to carbamazepine presenting with a leukemoid reaction, eosinophilia, erythroderma, and renale failure. Neurology 1989; 39: 436-438

${ }^{35}$ Stephan W, Parks R, Tempest B. Acute hypersensitivity pneumonitis associated with carbamazepine therapy. Chest 1978; 74: 463-464

${ }^{36}$ Taliercio C, Olney B, Lie J. Myocarditis related to drug hypersensitivity. Mayo Clin Proc 1985; 60: 463-468

${ }^{37}$ Braithwaite RF. A fatal case of agranulocytosis associated with „Tridione" therapy. Br Med J 1948; 1: 14

${ }^{38}$ Spielberg SP, Gordon GB, Blake DA et al. Predisposition to phenytoin hepatotoxicity assessed in vitro. N Engl J Med 1981; 305: 722 - 727

${ }^{39}$ Leeder JS. Mechanisms of idiosyncratic hypersensitivity reactions to antiepileptic drugs. Epilepsia 1998; 39: 8-16

${ }^{40}$ Riley R, Smith G, Wolf C et al. Human anti-endoplasmic reticulum autoantibodies produced in aromatic recognice rodent. CYP3A proteins and a similary regulated human P450 enzyme. Biochem Biophys Res Commun 1993; 191: $32-40$

${ }^{41}$ Aihara Y, Ito SI, Kobayashi Y et al. Carbamazepine-induced hypersensitivity syndrome associated with transient hypogammaglobulinaemia and reactiviation of human herpesvirus 6 demonstrated by real-time quantitative polymerase chain reaction. Br J Dermatol 2003; 149: $165-169$

${ }^{42}$ Hara H, Kobayashi M, Yokoyama A et al. Drug-induced hypersensivitity syndrome due to carbamazepine associated with reactivation of human herpesvirus 7. Dermatology 2005; 211: 159-161

${ }^{43}$ Hashimoto K, Yasukawa M. Tohyama M. Human herpesvirus 6 and drug allergy. Curr Opin Allergy Clin Immunol 2003; 3: 255-260

${ }^{44}$ Yates P, Stockdill G, McIntyre M. Hypersensitivity to carbamazepine presenting as pseudolymphoma. J Clin Pathol 1986; 39: 1224-1228

${ }^{45}$ Houwerzijl J, de Gast G, Nato JP et al. Lymphocyte-stimulation tests and patch tests in carbamazepine hypersensitivity. Clin Exp Immunol 1977; 29: $272-277$

${ }^{46}$ Hyson C, Sadler M. Cross sensitivity of skin rashes with antiepileptic drugs. Can J Neurol 1997; 24: 245-249

47 Shear NH, Spielberg SP. Anticonvulsant hypersensitivity syndrome. J Clin Invest 1988; 82: 1826-1832

${ }^{48}$ Mutschler E. Mutschler Arzneimittelwirkungen. 8. Aufl. Stuttgart: Wissenschaftliche Verlagsgesellschaft, 2002
Buchbesprechung

\section{Wirksame Hilfe bei Haarausfall. Ursachen erkennen, Lösungen finden. Tipps für einen selbstbewussten Alltag. Besser leben mit HAIRCOACHING}

Jenny Latz

Stuttgart: Trias, 2005. 173 S., 77 Abb., Kart. 14,95€,

ISBN 3-8304-3287-9

Nach dem ersten Ratgeber „Haar-Los. Der Ratgeber bei Haarproblemen“ der 1998 erschienen ist, hat Jenny Latz nun erneut einen gut lesbaren Patientenratgeber verfasst, der den Betroffenen hilft, mit diesem sehr schwierigen Thema umzugehen. Zum einen werden in verständlicher Form die unterschiedlichen Ursachen von Haarausfall besprochen, zum anderen aber, und das ist die Stärke dieses Buches, bis ins kleinste Detail Tipps und Tricks vermittelt, wie vor allem Frauen mit Haarverlust oder Haarlosigkeit umgehen lernen. Trotz aller gegenteiliger Versicherungen der Pharmaindustrie, ist die Patentlösung bei keiner Form des Haarausfalls gefunden. Die unterschiedlichen pharmakologischen und auch paramedizinischen Therapieansätze werden korrekt dargestellt. Dies geht bis zu so konkreten Tipps, wie ein Patient einen kompetenten „Haar-kundigen“ Dermatologen identifizieren kann: das lohnt sich auch für uns Ärzte einmal nachzulesen, wie unser Handeln empfunden wird. Gerade bei den schweren Formen des Haarausfalls wie der Alopecia areata totalis, der schweren androgenetischen Alopezie der Frau oder den vernarbenden Alopezien, kommt es auf ein vertrauensvolles Verhältnis zwischen Arzt und Patient an, damit die häufig frustranen Therapien richtig eingeschätzt und eingesetzt werden. Die psychischen Probleme, die mit Haarausfall verbunden sind, werden eingehend beleuchtet und aus der langen Erfahrung der selbst betroffenen Autorin dargestellt. Die unterschiedlichen gängigen Therapiemodalitäten, ob medikamentös oder kosmetisch werden für jede Alopezieform durchgesprochen - leider ohne Angabe von Literaturquellen, zum Teil auf Herstellerangaben beruhend. An diesem Teil des Buches merkt man sehr deutlich die Zielgruppe: Patienten, nicht Ärzte, denn für den fachkundig beratenden Arzt ist die Kenntnis der Primärquellen unverzichtbar, um nicht in die Gefahr zu kommen, der Pharmaindustrie das Wort zu reden. Der Leser muss sich darauf verlassen, dass die Informationen aktuell und korrekt zusammengestellt sind und kann bei Zweifeln ja stets uns Dermatologen zu Rate ziehen. Weiterhin findet sich in dem Buch eine Linkliste und Hilfen bei der Internet-Recherche. Den Abschluss bilden ein leider nicht ganz aktuelles Adressenverzeichnis und ein hilfreiches Stichwortverzeichnis.

H. Kurzen, Mannheim 